\title{
Time-of-day clocks and SKED: Techniques and applications
}

\author{
TIMOTHY F. ELSMORE \\ Walter Reed Army Institute of Research, Washington, D. C. 20012
}

\begin{abstract}
Modifications of SKED have been developed which incorporate a time-of-day clock into the basic SKED run-time systems. These clocks provide the experimenter with a means of recording the exact time of day at which events occur, as well as starting and stopping experiments and dumping data at specific times of the day. One type of clock is a hardware device that can be read to provide the number of minutes elapsed since midnight. A simple algorithm can be used to convert this value to hours and minutes. A second type of system maintains the current time by accumulating system clock ticks.
\end{abstract}

The SKED software system evolved as an attempt to replace traditional psychology laboratory instrumentation with a small general-purpose computer (Snapper \& Kadden, 1973). The system was written with traditionai experimental designs in mind, and thus features were included to optimize experiments involving running small groups or individual subjects for short sessions each day. The notational system that forms the basis for the SKED language, however, is considerably more general in scope (Snapper, Knapp, \& Kushner, 1970), and can easily describe procedures requiring more extended experimental sessions.

The present modifications to SKED arose from requirements to control experimental environments in which the subjects lived for the duration of the experiment. Control of behavior in continuous experimental environments is not fundamentally different from that required for shorter experimental sessions. However, it becomes necessary to have a means of synchronizing the experiment with the clock. While earlier versions of SKED could perform these functions, it was necessary to have the program for each experimental station keep track of the clock time and to have a means of initializing the time for each station. This involved considerable waste of computer time and memory, and led to the development of time-of-day clocks described in this paper, which could be incorporated into the SKED monitor and accessed as required by individual experiments.

Two different techniques have been developed for maintaining time-of-day information in SKED. Both systems perform the functions of maintaining locations in memory containing the current hour (0-23), the current minute (0-59), and the current 24-h time (0-2359). Assembly language subroutines (F3 routines) incorporated in the SKED monitor can be called from SKED programs to control program operation based on

Reprints, circuit diagrams, and listings are available from the author, Department of Medical Neurosciences, Walter Reed Army Institute of Research, Washington, D.C. 20012. any one of these three values. For example, sessions can be started and stopped at any desired time, houselights can be turned on and off, or data dumped exactly when required. Another subroutine allows the recording of the exact time in one of the recording counters of a SKED program. This has been used to record such things as the time at which a certain criterion for response acquisition is met and the exact times of day subjects earn food pellets. Finally, the time of day is dumped as part of the header line for every data dump, along with the current date, station number, number of counters, the subject number, and the experiment number. Thus, each dump is uniquely labeled, decreasing the possibility of confusion when the data are analyzed.

The first time-of-day clock is totally software and consists of a subroutine that is called with each tick of the SKED real-time clock $(100 \mathrm{~Hz})$. This routine updates the time locations, as well as incrementing the 0S/8 system date each midnight. Routines are available to run on any PDP- 8 computer or a PDP-8/E computer with extended arithmetic element. This system requires the system operator to initialize the time locations each time the SKED executive program is started.

The second type of clock uses a digital clock interfaced to the computer by a general-purpose interface card. ${ }^{1}$ The digital clock maintains a binary count of the number of minutes elapsed since midnight. This number is read by a subroutine each clock tick, and the hour, minute, 24-h time, and $0 \mathrm{~S} / 8$ date words updated as necessary. The time is also displayed on the front panel on seven-segment LED displays and in binary form on 12 single LEDs, primarily for trouble-shooting purposes. The clock includes circuitry for initializing the time and a battery back-up for maintaining accurate time even when the ac power is off. Thus, once the time is initialized, no time is required to keep it current. ${ }^{2}$ This clock was assembled from components costing less than $\$ 70$, and the interface board retails for $\$ 96.20$. A circuit diagram is available from the author.

These modifications to the SKED system are being incorporated into newer versions of the system. Earlier 
versions of the SKED system can also be modified to make use of either of the time-of-day clocks that have been described.

\section{REFERENCES}

SNAPPER, A. G., \& Kadden, R. M. Time sharing on a small computer based on a behavioral notation system. In $B$. Weiss (Ed.), Digital computers in the behavioral laboratory. New York: Appleton-Century-Crofts, 1973. Pp. 41-97.

SNAPPER, A. G., KNAPP, J. Z., \& Kushner, H. K.
Mathematical description of schedules of reinforcement. In W. N. Schoenfeld (Ed.), The theory of reinforcement schedules. New York: Appleton-Century-Crofts, 1970. Pp. 247-275.

\section{NOTES}

1. Part 26-DE-8, available from Douglas Electronics Inc., 718 Marina Boulevard, San Leandro, California 94577.

2. The clock was designed and built by Maurice Swinnen of the Department of Microwave Research, Walter Reed Army Institute of Research. 